visit and 1 to 4 follow up visits, with additional visits for flares. Peripheral blood mononuclear cells (PBMC) were stained with a dead cell marker and antibodies to CD19, CD27, and IgD. Antibody binding capacity (ABC) of CR1 and CR2 on live bulk and gated B cells was determined using quantitative flow cytometry (Bang Labs). The frequency of live gated B cells was determined. Disease activity was measured using SLEDAI. Statistical analyses were performed using R. To determine associations between the SLEDAI scores and gate/quadrant signals, negative binomial mixed effect models were fitted using the glmmADMB package. A random effect intercept was included to account for different individual baseline SLEDAI scores. The gate/ quadrant signals, in terms of $\mathrm{ABC}$ and percentages, were the independent variables. Adjustments were made for age, prednisone dose, and, where relevant, bead lot. To account for multiple testing in bulk B cells, a conservative Bonferroni adjustment was used, whereas in gated cells, the p-values were adjusted to control the false discovery rate according to Benjamini-Hochberg. A p-value of $\leq 0.05$ was considered significant.

Results CR1 and CR2 ABC on bulk B cells and on $\mathrm{IgD}+\mathrm{CD} 27-, \operatorname{IgD}+\mathrm{CD} 27+$, and $\operatorname{IgD}-/ \mathrm{CD} 27$ +gated $\mathrm{B}$ cells was negatively associated with lupus disease activity, whereas there was no association between CR1 and CR2 $\mathrm{ABC}$ on IgD-/CD27- B cells or frequency of any of these gated populations and disease activity (table 1 and data not shown).

\begin{tabular}{|c|c|c|c|c|}
\hline B cells & Independent variable & Estimate & Standard Error & Adjusted $p$ value \\
\hline Bulk & CR1 ABC & $-3.07 \mathrm{E}-05$ & $1.09 \mathrm{E}-05$ & 0.018771099 \\
\hline Bulk & CR2 ABC & $-3.49 \mathrm{E}-05$ & $9.77 \mathrm{E}-06$ & 0.001413636 \\
\hline $\operatorname{lgD}+C D 27-$ & CR1 ABC & $-1.47 \mathrm{E}-05$ & $6.50 \mathrm{E}-06$ & 0.031872055 \\
\hline $\lg D+C D 27-$ & CR2 ABC & $-3.06 \mathrm{E}-05$ & $9.65 \mathrm{E}-06$ & 0.005642294 \\
\hline $\lg \mathrm{D}+\mathrm{CD} 27+$ & CR1 ABC & $-1.74 \mathrm{E}-05$ & $6.32 \mathrm{E}-06$ & 0.013441797 \\
\hline $\lg \mathrm{D}+\mathrm{CD} 27+$ & CR2 ABC & $-2.46 \mathrm{E}-05$ & $7.88 \mathrm{E}-06$ & 0.005642294 \\
\hline $\operatorname{lgD}-\mathrm{CD} 27+$ & CR1 ABC & $-2.77 \mathrm{E}-05$ & $8.63 \mathrm{E}-06$ & 0.005642294 \\
\hline $\operatorname{lgD}-\mathrm{CD} 27+$ & CR2 ABC & $-4.25 \mathrm{E}-05$ & $1.21 \mathrm{E}-05$ & 0.003641553 \\
\hline $\lg D-C D 27-$ & CR1 ABC & $-2.53 \mathrm{E}-07$ & $2.48 \mathrm{E}-06$ & 0.918673439 \\
\hline $\operatorname{lgD}-C D 27-$ & CR2 ABC & $-3.72 \mathrm{E}-06$ & $1.10 \mathrm{E}-05$ & 0.785689588 \\
\hline
\end{tabular}

Conclusions Both CR1 and CR2 levels are negatively associated with lupus disease activity in defined B cell subsets. Although this study does not prove causality, these data suggest that altered levels of these receptors on specific B cell subsets may predict disease flare or be associated with disease remission.
Acknowledgements This work was supported by R01AI070304, K24AI078004, and the Lupus Research Institute.

\section{II-09 IMMUNOLOGIC PROPERTIES OF CUTANEOUS LUPUS ERYTHEMATOSUS (CLE) PATIENTS REFRACTORY TO ANTIMALARIALS COMPARED TO PATIENTS THAT RESPOND TO ANTIMALARIALS}

$1^{1,2}$ Krisha Desai, 1,2 Majid Zeidi, $1,2,3$ Hee Joo Kim, ${ }^{1,2}$ Victoria P Werth*. ${ }^{1}$ Corporal Michael J.
Crescenz VAMC, Philadelphia, PA, USA; ${ }^{2}$ Department of Dermatology, University of
Pennsylvania, Philadelphia, PA, USA; ${ }^{3}$ Department of Dermatology, Gil Medical Center,
Gachon University College of Medicine, Incheon, Korea

\subsection{6/lupus-2018-Ism.108}

Background Two major therapies for cutaneous lupus erythematosus (CLE) are the antimalarials, hydroxychloroquine (HCQ) and quinacrine (QC). HCQ is often the first-line therapy for CLE, but only half of patients show a response to it. While some of the patients that do not initially respond to HCQ benefit from the addition of QC, there is a subset of patients that are refractory to both antimalarials. Refractoriness poses a huge challenge because these patients will often continue to have active disease when they are initially started on antimalarials. To better characterize these refractory patients, we investigated the immunologic characteristics of patients that respond to antimalarials versus those that do not.

Methods CLE patients were classified as HCQ-responders, HCQ/QC-responders, or HCQ/QC-nonresponders. Immunohistochemistry was used to characterize the inflammatory cell composition and cytokine expression in lesional skin biopsies from patients. Total RNA was extracted from these biopsies to analyze specific gene signatures. The patient's CLASI score - a measure of disease activity - at the time of the biopsy was also determined.

Results Immunohistochemistry showed that myeloid dendritic cells (mDCs) were significantly higher in HCQ/QC-responders compared to HCQ-responders and HCQ/QC-nonresponders, while plasmacytoid dendritic cells, neutrophils, macrophages, and autoreactive $\mathrm{T}$ cells did not differ significantly among the three groups. The HCQ/QC-nonresponder group was distinct from the other groups in that their CLASI scores did correlate positively with the number of macrophages $(p<0.05$, figure $1)$. Staining also showed that IL-22 expression was significantly higher in HCQ/QC nonresponders versus the HCQ or HCQ/QC- responders while IL-17 expression was not significantly different between the responders and nonresponders. Analyzing the mRNA expression demonstrated a high type I IFN signature (LY6E, OAS1, ISG15, MX1) in HCQ-
A

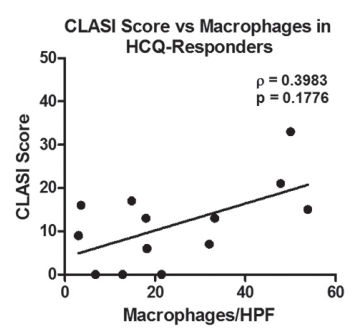

B

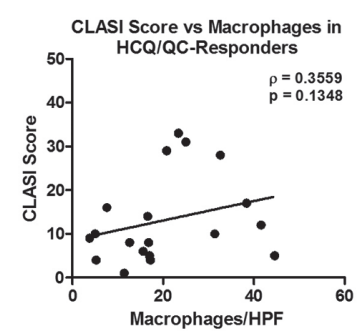

C

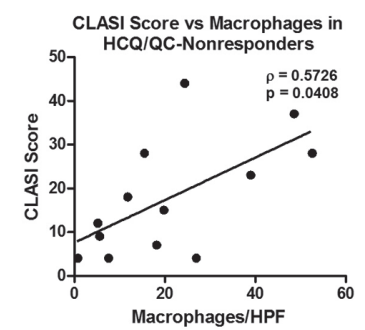


responders but a low type I IFN signature and higher TNFalpha expression in both HCQ/QC-nonresponders and HCQ/ QC-responders.

Conclusions An increased number of $\mathrm{mDCs}$ may contribute to HCQ-refractoriness and predict a better response to treatment with both HCQ and QC but do not contribute to HCQ/QCrefractoriness. The significant correlation between macrophages and CLASI scores in the HCQ/QC-nonresponders, a finding not seen in either HCQ or HCQ/QC-responders, may also indicate that macrophages are more involved in antimalarialrefractory skin disease. The difference between the responders and nonresponders is further confirmed by the cytokine staining and mRNA expression. Our data is an initial step in determining the activation pathways that account for the lack of response to antimalarials.

Acknowledgements This work was supported by National Institutes of Health [K24 AR002207] and Veterans Affairs Merit Review [I01B $\times$ 000706] to Dr. Victoria Werth.

\section{II-10 ROLE OF MACROPHAGE-DRIVEN AUTOINFLAMMATION IN SLE}

${ }^{1}$ Shuhong Han, ${ }^{1}$ Haoyang Zhuang, ${ }^{2}$ Pui Lee, ${ }^{1}$ Lijun Yang, ${ }^{1}$ Westley H Reeves*. ${ }^{1}$ University of Florida, Gainesville, FL, USA; ${ }^{2}$ Boston Children's Hospital, Boston MA, USA

\subsection{6/lupus-2018-Ism.109}

Background Although SLE is a prototype of autoimmune (T cell/B cell-mediated) disease, there is increasing evidence implicating myeloid cells in its pathogenesis. We examined the role of macrophages (MФ) in human SLE and the pristane-induced lupus model.

Methods Tissues of SLE patients and mice with pristane-induced lupus were examined by double immunohistochemistry. MФ were analyzed by flow cytometry, phagocytosis assay, real-time PCR, Seahorse assay, and RNA-Seq. Mice were injected daily with the liver X receptor (LXR) agonist T0901317.

Results SLE patients' bone marrow contained numerous activated caspase- $3^{+}$apoptotic cells located outside of МФ. In contrast, in leukemia patients undergoing bone marrow ablation prior to transplantation, all caspase $-3^{+}$cells were inside of $M \Phi$, suggesting that lupus is associated with impaired clearance of apoptotic cells. M $\Phi$ in pristane-lupus took up fluorescently-labeled apoptotic cells poorly compared with controls. Studies in pristane-treated knock-out mice indicated that the induction of diffuse alveolar hemorrhage (DAH), a serious pulmonary complication of lupus, required opsonization of dead cells in the lung by 'natural' IgM (DAH absent in $\mu$ MT mice, restored by infusion of $\operatorname{IgM}$ ), C3, and $\mathrm{C} 3 \mathrm{~b}$ receptors (absent in C3-/- and CD18-/- mice, prevented by complement depletion with cobra venom factor). DAH also was prevented by MФ depletion (clodronate liposomes) but not neutrophil depletion (anti-Ly6G). MФ in pristane-induced lupus exhibited features of classical activation (impaired phagocytosis of apoptotic cells, high glycolysis, low oxidative phosphorylation, increased HIF1 $\alpha$ expression, M1 surface markers, and TNF $\alpha$ production), whereas control MФ from mice treated with mineral oil (do not develop DAH) were M2-like, with high phagocytic activity, low glycolysis, high OxPhos, increased LXR $\alpha$ expression, M2 surface markers, and IL-10 production. In both pristane-induced lupus MФ and SLE patients' monocytes, we found low levels of the LXR $\alpha$-regulated reverse cholesterol transporter ABCA1. We hypothesized that it might be possible to treat DAH by 're-polarizing' M $\Phi$ using a synthetic LXR agonist. Consistent with that idea, pristane-treated mice receiving T0901317 did not develop DAH and MФ from mice receiving T0901317 exhibited an M2-like surface phenotype and decreased TNF $\alpha$ production compared with controls.

Conclusions Our data suggest that lupus-associated DAH is partly an autoinflammatory response caused by sluggish clearance of apoptotic cells by M1-like $\mathrm{M} \Phi$ and that certain clinical manifestations (e.g. DAH) can be treated by repolarizing $M \Phi$ using an activator of the transcription factor $\operatorname{LXR} \alpha$. Low levels of LXR-driven ABCA1 expression also may have implications for the therapy of atherosclerosis in SLE.

\section{II-11 SIGLEC-1 MACROPHAGES AND THE CONTRIBUTION OF IFN TO THE DEVELOPMENT OF AUTOIMMUNE CONGENITAL HEART BLOCK}

Robert Clancy*. New York University Langone Medical Center, New York, New York

\subsection{6/lupus-2018-Ism.110}

Background Given that diseases associated with anti-Ro such as SLE and Sjögren's syndrome associate with an upregulation of type I interferons, recent attention has focused on a potential role for IFN in the pathogenesis of congenital heart block $(\mathrm{CHB})$. Based on the consistent demonstration of macrophages and multinucleated giant cells in areas of injury, it is relevant that Sialic Acid Binding Ig Like Lectin 1 (SIGLEC1), a receptor on monocytes/macrophages is upregulated by IFN. Functionally, Siglec-1 expressing macrophages might play an important role as effector cells in fibrosis. Accordingly, this study leveraged both autopsy tissue and freshly isolated macrophages from a fetal heart dying with $\mathrm{CHB}$ to address whether IFN $-\alpha$ contributes to the pathogenesis of $\mathrm{CHB}$ by regulating activated macrophages in affected cardiac tissue.

Methods Three approaches were taken to evaluate Siglec-1 expression. Transcriptomic analysis was performed on macrophages freshly isolated from a fetal heart dying with $\mathrm{CHB}$ at 19 weeks and a heart from an otherwise healthy electively terminated fetus using (DAPI negative cells with isolation by flow using antibodies to CD45). Immunohistochemistry was performed on another fetal heart dying with CHB. In vitro experiments utilized cultured healthy human macrophages transfected with anti-SSA/ Ro- associated ssRNA as a proxy for the in vivo conditions.

Results Transcriptomes of the two hearts for each isolated leukocyte fraction were compared. By following 213 IFN inducible genes, there was enrichment of targeted transcripts in CHB vs control $(p=0.0001)$ and SIGLEC1, which was 200fold more abundant in $\mathrm{CHB}$ vs control and ranked among the top three differentially expressed candidates. In another fetal heart dying with $\mathrm{CHB}$, Siglec1 staining as detected by antibody HPA053457 was prominent in areas of injury. By morphology, the two cell types expressing Siglec 1 were macrophages and dendritic cells. In vitro experiments were performed in accordance with previous laboratory work, in which a model of anti-SSA/Ro-associated injury exploits macrophages stimulated with the ssRNA component (hY3) of the SSA/Ro immune complex. IFN inducible genes (15 transcripts) were among the 30 most highly upregulated genes in hY3 stimulated conditions and SIGLEC1 was two-fold more abundant in $\mathrm{CHB}$ vs control. Given the enrichment of type I IFNresponsive genes in the macrophage transcriptome, a WISH cell line was selected to evaluate supernatants from 